\title{
Der 1-Minuten-Aufstehtest bei COPD
}

\author{
Bei Patienten mit einigermaßen intakten Kniegelenken kann der 1-Minuten-Aufstehtest \\ aufwändigere Tests zur Bestimmung der pulmonalen Leistungsfähigkeit bei COPD ersetzen.
}

\begin{abstract}
Patienten aus zwei pneumologischen Rehabilitationskliniken in der Schweiz wurden bei Aufnahme und Entlassung gebeten, innerhalb einer Minute so oft wie möglich von einem normalen Stuhl aufzustehen, ohne die Arme zu benutzen. Körperlich beeinträchtigte Patienten wurden ausgeschlossen. 48 Teilnehmer beendeten die Studie, bei der auch verschiedene Atemparameter gemessen wurden.

Die Teilnehmer verbesserten sich von 15,3 $\pm 6,2$ auf 19,8 \pm 7,8 Wiederholungen. Im ebenfalls durchgeführten 6-Minuten-Gehtest drückte sich das in einer Verlängerung der Gehstrecke von 286,5 $\pm 127,6$ auf $367,2 \pm 130,8 \mathrm{~m}$ aus
\end{abstract}

Des Weiteren wurden 203 Teilnehmer einer anderen Studie einbezogen, die ebenfalls den 1-Minuten-Aufstehtest und den 6-Minuten-Gehtest gemacht und COPD-Fragebögen ausgefüllt hatten. Bei ihrer Aufnahmeuntersuchung machten sie in kurzem Abstand zwei 1-Minuten-Aufstehtests, um die Reproduzierbarkeit zu ermitteln. Es ergab sich eine geringfügige Steigerung um eine Wiederholung. Die Intratest-Korrelationskoeffizienten betrugen 0,93 für den Lerneffekt und 0,99 für die Reliabilität. $\mathrm{O}_{2}$-Verbrauch, $\mathrm{CO}_{2}$-Ausstoß, Atem- und Herzfrequenz waren im 1-Minuten-Aufstehttest und im 6-Minuten-Gehtest vergleichbar.
- Crook S et al. A multicentre validation of the 1-min sit-tostand test in patients with COPD. Eur Respir J. 2017;49:1601871

\section{KOMMENTAR}

Der 1-Minuten-Aufstehtest ist so einfach, dass er von jedem Patienten sofort verstanden wird und in jeder Praxis ohne jede Vorbereitung und auch vom Praxispersonal durchführbar ist. Er erbringt ebenso zuverlässige Informationen über die körperliche Leistungsfähigkeit wie aufwändigere Verfahren. Er kommt allerdings nicht für alle Patienten infrage, da eine geschwächte Oberschenkelmuskulatur sowie Beeinträchtigungen des Kniegelenks das Ergebnis erheblich verzerren können.

Prof. Dr. med. H. S. FüeßI

\section{Druckdolenter Strang unterhalb der Brustwarze}

Ein 53-jähriger Mann mit Hypertonie und Diabetes mellitus hatte seit vier Tagen Schmerzen in der rechten Brustwarze. An eine Verletzung konnte er sich nicht erinnern, es lag kein Fieber vor, auch hatte er keinen Ausfluss aus der Brustwarze bemerkt. Bei der körperlichen Untersuchung erkannte man einen druckdolenten subkutanen Strang, der an der rechten vorderen Axillarfalte begann und sich an der Brustwarze vorbei bis in das rechte Hypochondrium erstreckte. Beim Anheben des rechten Arms über die Horizontale trat der Strang deutlicher hervor. Die Dopplersonografie zeigte eine nicht komprimierbare thorakale epigastrische Vene, die echogenes Material enthielt. Dieser Befund war typisch für eine oberflächliche Venenthrombose. Hinweise auf tiefe Beinvenenthrombosen lagen nicht vor.

Die oberflächliche Thrombophlebitis einer Vene an der seitlichen Thorax- bzw. Bauchwand ist bekannt als Mondorsche Erkrankung. Sie ist typischerweise gutartig und selbstlimitierend und kommt bei Frauen häufiger als bei Männern vor. Zu den Risikofaktoren gehören Traumen, Operationen im Bereich der Brust und heftige körperliche Aktivität. Gelegentlich wurde die Erkrankung auch bei Patientinnen mit Mammakarzinom beschrieben. Die Behandlung besteht in der Verabreichung nicht-steroidaler Antiphlogistika. Im vorliegenden Fall bildete sich die Veränderung innerhalb von sechs Wochen zurück und trat im Verlauf des nachfolgenden Jahres nicht mehr auf.

Prof. Dr. med. H. S. FüeßI

- Vijayalakshimi A, Anand S. Mondor's disease. N Engl J Med. 2017;376:e47

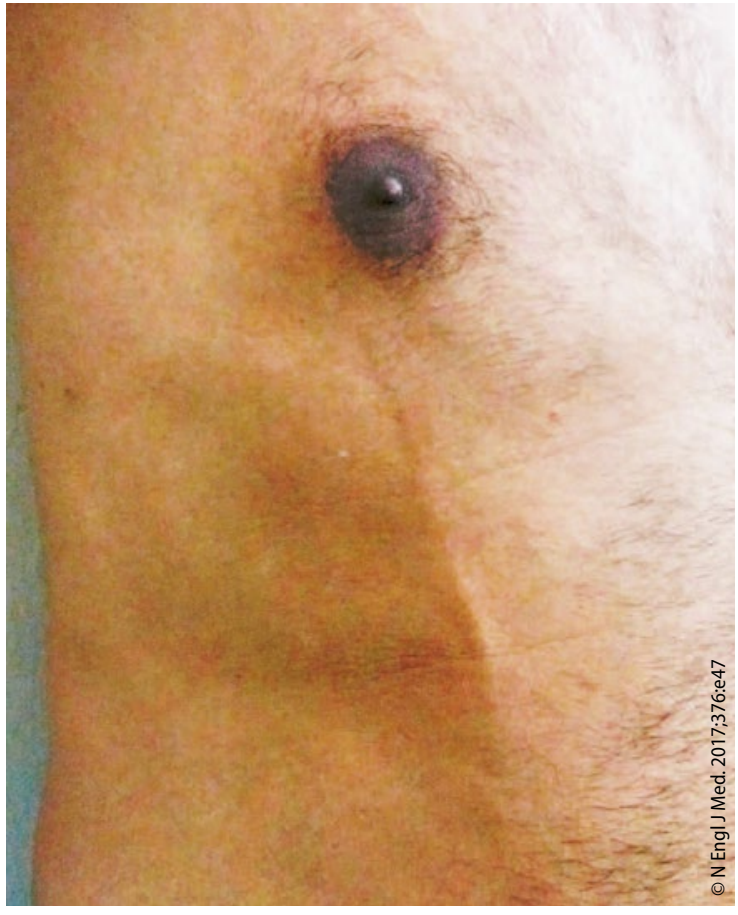

Subkutaner Strang an der Vorderseite des Torsos. 